\title{
Aerodynamics of Flapping Wing at Low Reynolds Numbers: Force Measurement and Flow Visualization
}

\author{
Abhijit Banerjee, Saurav K. Ghosh, and Debopam Das \\ Unsteady Aerodynamics Laboratory, Department of Aerospace Engineering, IIT Kanpur 208016, India \\ Correspondence should be addressed to Debopam Das, das@iitk.ac.in
}

Received 31 January 2011; Accepted 8 March 2011

Academic Editors: M. Ali and P. Zhang

Copyright (๑) 2011 Abhijit Banerjee et al. This is an open access article distributed under the Creative Commons Attribution License, which permits unrestricted use, distribution, and reproduction in any medium, provided the original work is properly cited.

\begin{abstract}
Flow field of a butterfly mimicking flapping model with plan form of various shapes and butterfly-shaped wings is studied. The nature of the unsteady flow and embedded vortical structures are obtained at chord cross-sectional plane of the scaled wings to understand the dynamics of insect flapping flight. Flow visualization and PIV experiments are carried out for the better understanding of the flow field. The model being studied has a single degree of freedom of flapping. The wing flexibility adds another degree to a certain extent introducing feathering effect in the kinematics. The mechanisms that produce high lift and considerable thrust during the flapping motion are identified. The effect of the Reynolds number on the flapping flight is studied by varying the wing size and the flapping frequency. Force measurements are carried out to study the variations of lift forces in the Reynolds number (Re) range of 3000 to 7000 . Force experiments are conducted both at zero and finite forward velocity in a wind tunnel. Flow visualization as well as PIV measurement is conducted only at zero forward velocity in a stagnant water tank and in air, respectively. The aim here is to measure the aerodynamic lift force and visualize the flow field and notice the difference with different Reynolds number (Re), and flapping frequency $(f)$, and advance ratios $\left(J=U_{\infty} / 2 \phi f R\right)$.
\end{abstract}

\section{Introduction}

With the advent in the progressive research for the application and fabrication of autonomous MAVs (micro aero vehicles) flapping mode flight is largely and widely studied in the past couple of decades. The applications of such MAVs can be enormous in terms of surveillance, monitoring, tracking, and other defence purposes in rough terrains and human restricted hazardous environments. There have been significant advances in the fixed wing or rotary wing-type MAVs. Another development is the onset of the MAVs with flapping wings type. The edge of this mode of flying type comes in terms of efficiency, maneuverability, and wide range of speed, which is perhaps the reason of its presence in nature among millions of species of birds and insects. However, this flapping wing flight is much more complex in terms of unsteadiness and three-dimensional nature, which cannot be summed up by conventional aerodynamics alone. Biologist have studied and produced the kinematic details of the various species giving fluid dynamist a grasp in the distinction of zones or behavior of flapping flight among species and their inherent traits. Some progress have been made using quasisteady and unsteady aerodynamics of simple flapping kinematics $[1,2]$, but the forces and control over them encountered is far more complicated owing to its dependence on wide array of aerodynamic mechanisms to take off, maneuver, maintain steady flight, and for landing [3].

From the experimental aspect a lot of study has been conducted in order to understand the flow physics of such flight and to generate the forces of required pattern with the chosen kinematics. The same has been achieved by smoke flow visualization over flapping wing in a wind tunnel [3] for a chosen kinematics and by PIV [4] for the dynamics of these vortex dominated flows [5-7]. Significant observations have been made in identifying the important governing parameters like Reynolds number $(\mathrm{Re})$ range, advance ratio $(J)$ and phenomena like the leading edge vortex domination $[4,8]$ of these flights hindering the flow separation, wake capture, clap and fling [8], and so on. Based on earlier studies 


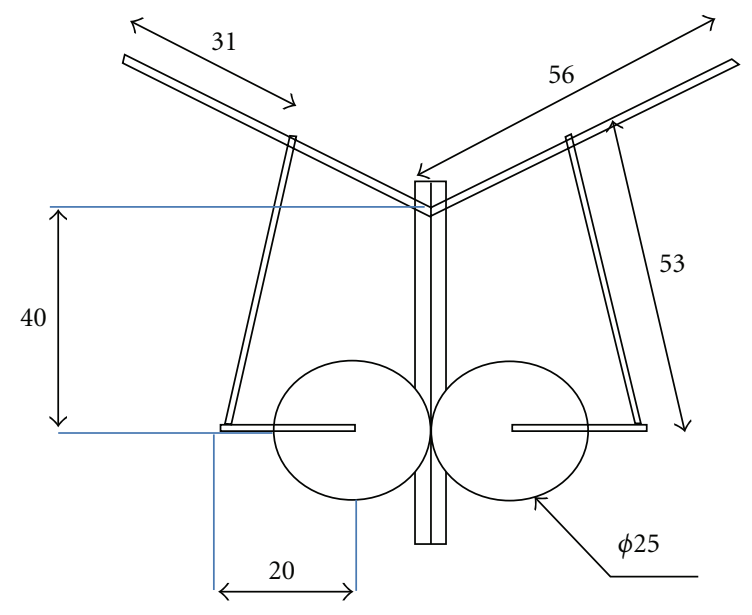

FIGURE 1: Schematic view of model (all units in $\mathrm{mm}$ ).

flapping flight can be broadly divided into two categories: quasisteady for $J>1$ and unsteady flight for $J<1$ [9]. While flights of large birds can be approximately explained using quasisteady theory, the insects' and small bird's flight are highly unsteady. Further references of the subject can be obtained from [2-17].

\section{Experimental Setup}

The aim of the experiments conducted is to understand the nature of flapping flight through the flow field visualization and measurement of aerodynamic forces generated in the flapping process. Force measurements are performed to encounter the unsteady forces generated while flapping. Apart from this, visualization study is performed for the model inside water to understand the vortical structures and their interaction in a better way. PIV experiments are carried out in air with the same model but with different wing shape. The motive is mainly to understand the underlying flow physics of insect flight thus focusing on totally unsteady regime.

\section{Experimental Model}

The model simulates flapping motion with a 4-bar quickreturn mechanism as shown in Figure 1. It has one degree of freedom of flapping with time ratio of $1: 1.3$, where downstroke is slower. The reason to choose a flapping mechanism with a relatively larger downstroke time is based on the observed flight patterns of flying animals with advance ratio $J<1$ such as bats, butterflies and certain other insects. Usually the maximum time ratio is $1: 2$, and it varies with the speed of flight and hence species of flier [10]. The wing leading edge is glued to the flapping linkage arm with which it flaps as shown in Figure 1. The free end of the wing deflects a little during flapping motion adding to the feathering effect. The bigger the wing the more feathering it undergoes. For the wings that are considered, the feathering angle that comes into play is small. But a little change in feathering angle also changes the dynamics of the force generation considerably as shown in results. Force measurements are carried out for various wings, and flow visualization is performed with a similar wing exhibiting the same Re range inside water.

\section{Force Measurement}

The lift force measurements are carried out in a closed circuit wind tunnel. The schematic sketch of the setup is shown in Figure 2. The tunnel has a contraction ratio of $11: 1$ and a working cross-section of $600 \mathrm{~mm} \times 900 \mathrm{~mm}$. Minimum operating speed of the tunnel is $1 \mathrm{~m} / \mathrm{s}$ and maximum speed is $20 \mathrm{~m} / \mathrm{sec}$. The turbulence intensity is about $0.5 \%$ at minimum speed and $0.2 \%$ at maximum speed. A 3-component platform balance is used for force measurements. The minimum resolution of the transducer is $0.01 \mathrm{gm}$. The least force measured is $0.3 \mathrm{gms}$ which makes the maximum uncertainty of the force measurement to be $3.3 \%$. Lift variation is the primary concern in this study, and is only measured. Quarter elliptical shaped wings (Figure 4(a)) of various sizes are chosen for the force experiments.

Two kinds of tests are performed on the model; Hovering tests, where forward velocity $\left(U_{\infty}\right)$ is zero and Flight tests, which has a nonzero $U_{\infty}$ and simulates flight through a medium. Both these tests are important in order to determine the difference in the nature of lift generation in the mentioned conditions.

\section{Flow Visualization}

To understand the complex unsteady mechanism of unusually high force generation through flapping wing, flow visualization experiments are carried out. Experiments have been carried out in a water tank, with size $(150 \mathrm{cms} \times$ $50 \mathrm{cms} \times 75 \mathrm{cms}$ ). Sodium fluorescent dye has been used for tracing the fluid path. Flow features of such unsteady flow are usually complex and dominated with vortical structures. Efforts have been made to visualize such vortex dominated flow and understand its role in flapping flight by injecting dye at suitable positions over the wing. The amount of dye injected and the location of ejection comes from hit and trial to get a good visualization.

All the visualization experiments are conducted in a water tank made of $6 \mathrm{~mm}$ glass and Perspex sheets. A light sheet is used to illuminate a particular plane of the flow field in study. A video camera (SONY DCR-VX2100E PAL) ( 25 frames/s) is mounted on top of the setup in such a way that it is perpendicular to the light sheet and captures the entire phenomenon in that plane. The schematic sketch is shown in Figure 3. The flapping wing mechanism is placed on the tank in such a way that the wing is completely immersed in water, even at the end stroke position.

The frequency of flapping at which experiments are performed is $0.46 \mathrm{~Hz}$ and $0.7 \mathrm{~Hz}$. The model is placed upside down such that the motor can be kept outside the water level. A light sheet is used to illuminate a particular plane and flow field is visualized using a camera perpendicular to this plane. Shape and size of single-wing model used for flow visualization is shown in Figure 5(a). The wing is a triangular wing with rounded edge. For flow visualization the wing 


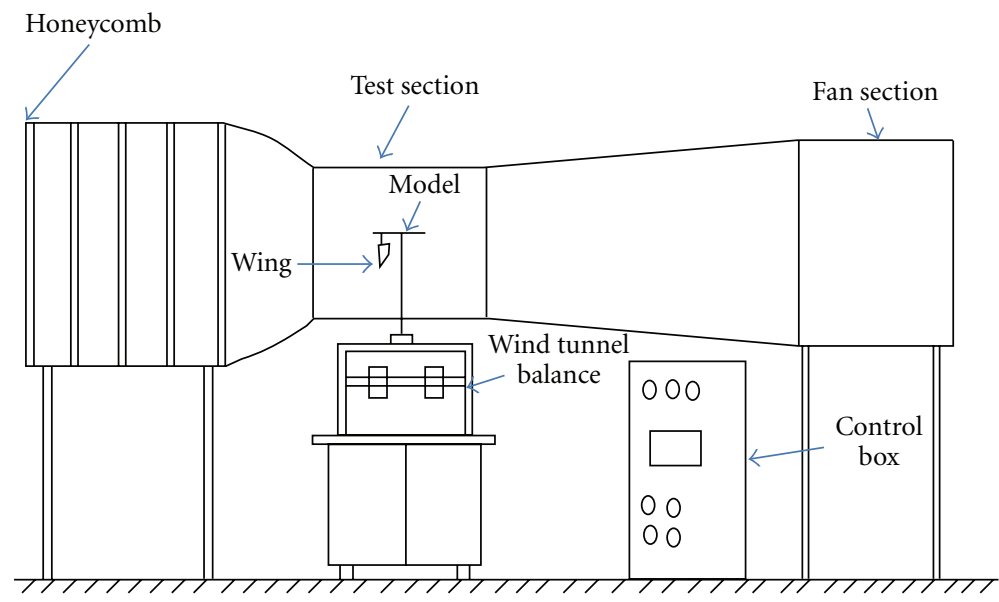

FIGURE 2: Experimental setup for aerodynamic force measurement.

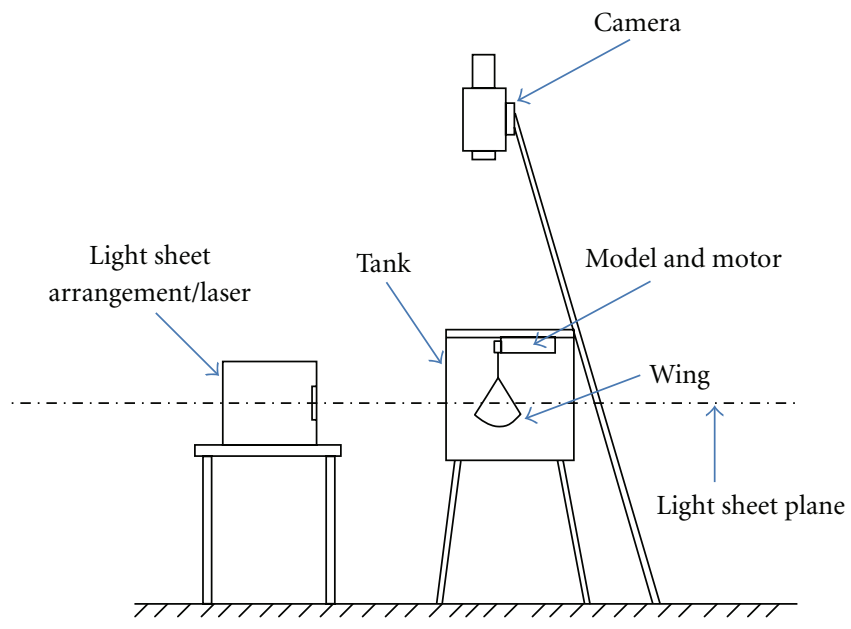

FIGURE 3: Diagrammatic representation of the flow visualization setup (side view).

shape is chosen like this to have a minimum interference of the LEV with the free surface. The result presented here belongs to the flapping frequency of $0.46 \mathrm{~Hz}$. The total angle of flapping for this wing is $60^{\circ}$ and the wing is started from a position $10^{\circ}$ away from complete clap (Figure 5(b)).

\section{PIV Setup}

PIV experiments are conducted in air on the same model but using a different wing (Figure 4(b)) to observe the similar nature of flowfield and the phenomena involved with the same kinematics of the wing. A double pulsed Nd: YAG laser (Quanta system, $200 \mathrm{~mJ} /$ pulse, $10 \mathrm{~Hz}$ max frequency) is used for illuminating the flow field. The time between the two laser pulses is kept as $978 \mu \mathrm{s}$. The size of the seeding particles is $\approx 1 \mu \mathrm{m}$. A Nanosense Mk III ( 1000 frames/sec, $1280 \times$ 1024 resolution) cameras is used for capturing the particle images. Laser and camera are synchronized by a timer box. Experiments are performed in a closed glass chamber for making the smoke and the seeded particles stay uniformly over the laser sheet of light. Measurements are carried out at different chord cross-sections of a butterfly-shaped wing (Figure 4(b)). The flapping frequency is kept constant during experiments at different sections. The velocity and vorticity variations of the flow field are calculated for different experiments using Dynamic Studio software from DANTEC dynamics with a $50 \%$ overlap in an interrogation area of $32 \times 32$ pixels of the camera.

The calibration for the PIV experiments is done by focusing the camera on a calibration target plate $(200 \mathrm{~mm} \times$ $200 \mathrm{~mm}$ ). The laser sheet is aligned with the plane of the calibration plate. The model is kept accordingly keeping in view that the measurement plane is the same where the laser sheet and the camera both are focused.

\section{Results and Discussion}

7.1. Force Results. Figure 6 shows the lift variation of an elliptical wing $(a=6.35, b=3.175)$ at $f=7.5 \mathrm{~Hz}$ at $J=0$, where $t / T=0$ denotes the start of downstroke. It is observed that due to a faster upstroke the peak negative force generated is also higher. However, as this negative peak stays for a shorter duration the net lift in the whole cycle is still positive. Figure 7 shows the variation of averaged lift or net lift generated with frequency for different wings mounted on the model for $U_{\infty}=0$. For a particular wing size as the frequency increases, lift force increases. It can be observed that the smallest size wing $(a=5.08, b=2.54)$ is producing more lift as it is flapping at highest frequency compared to the other wings. Thus, size of wing within the range studied is not an important factor for generating lift. It is the frequency which is more critical. Figure 8 shows the variation of rotational lift coefficient with Reynolds number. Reynolds number here is defined in terms of tip-speed $\left(V_{t}\right)$ whereas with forward velocity Reynolds number is defined in terms of $U_{\infty}$. For the smallest wing the $C_{L}$ value is found to be larger compared to other three wings even at the same frequency. 


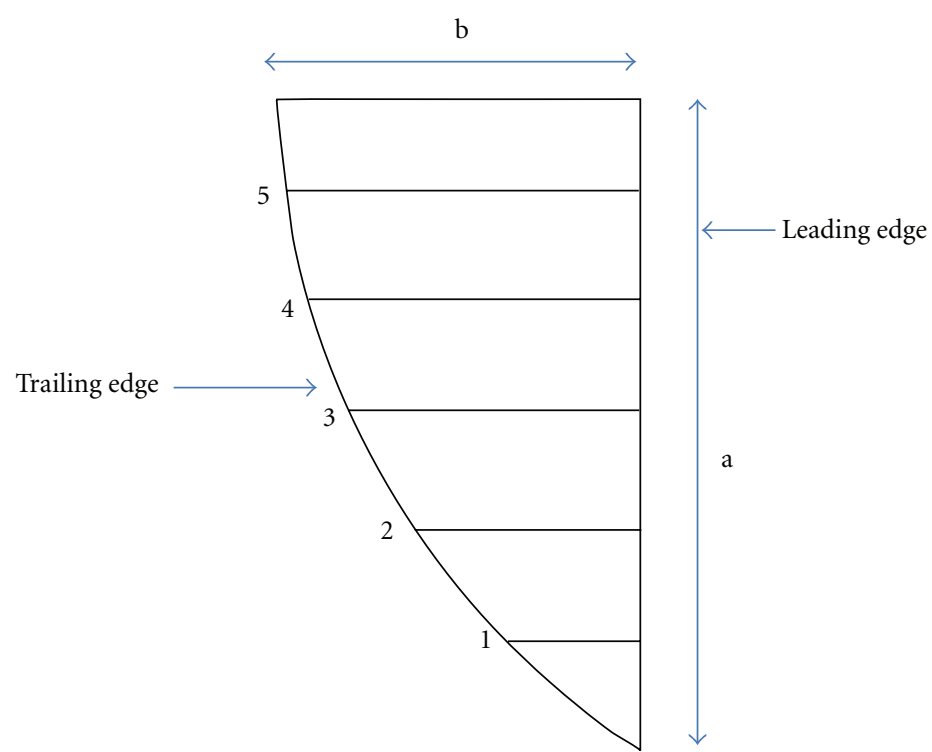

(a)

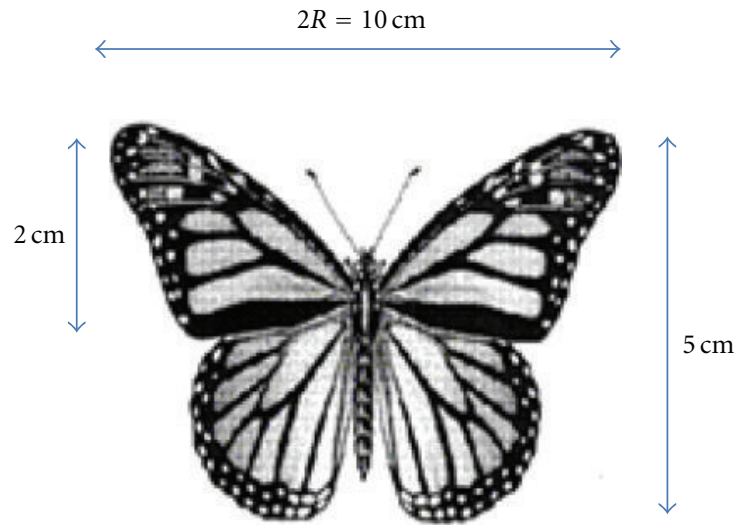

(b)

FIGURE 4: (a) Quarter elliptical wing. (b) Butterfly shaped wing of Mylar membrane.

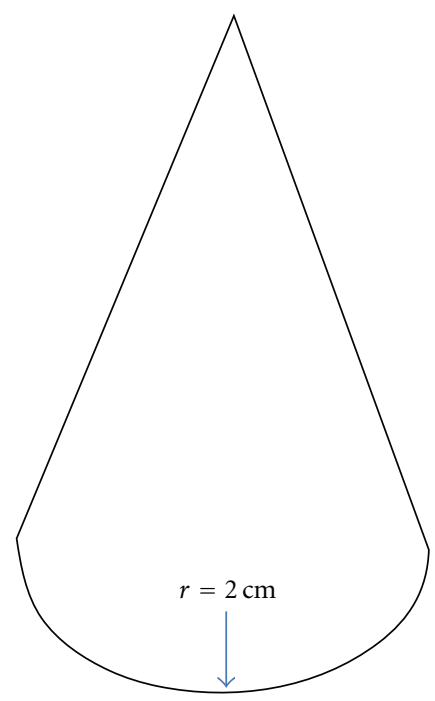

(a)

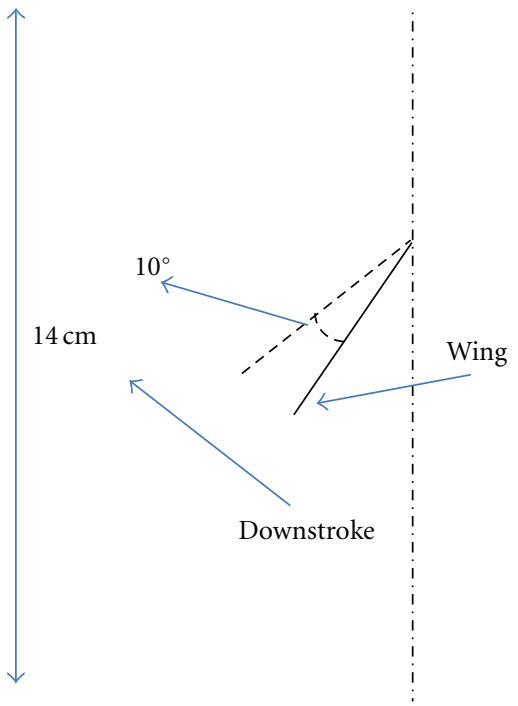

(b)

FIGURE 5: (a) Dimensions of the symmetrical wing. (b) wing at the starting position.

In the experiments conducted all the wings are glued by their leading edges to the arm of the flapping linkage. Thus the trailing edge hangs free. The longer the chord more the chordwise flexibility it is going to achieve. The constraint on the chordwise flexibility may be the cause of the large deviation in the $C_{L}$ of the small wing.

From Figures 9(a) and 9(b) it can be seen that the lift generated by the model decreases as $J$ increases, irrespective of the forward velocity or the wing size. Comparison of Lift versus advance ratio $(J)$ data for the other wings shows the same trend. Hence, with more unsteadiness in the flow a higher lift coefficient can be obtained. Again, for the same forward velocity $J$ decreases with increasing frequency showing a higher lift verifying the earlier observation that lift increases with frequency and frequency plays a critical role as it defines the rotational $\mathrm{Re}_{\text {tip }}$. Similar nature of lift versus $J$ has also been notified in the past for other kinematic pattern, different wing geometry and different Re [9].

The lift force comprises of two components in forward velocity cases. One is the circulatory component that is due to the forward velocity and the resulting effective angle of attack caused further by flapping. The other is the added mass component that arises due to force exerted by the enveloped air mass accelerating with the wing itself. Normally without 


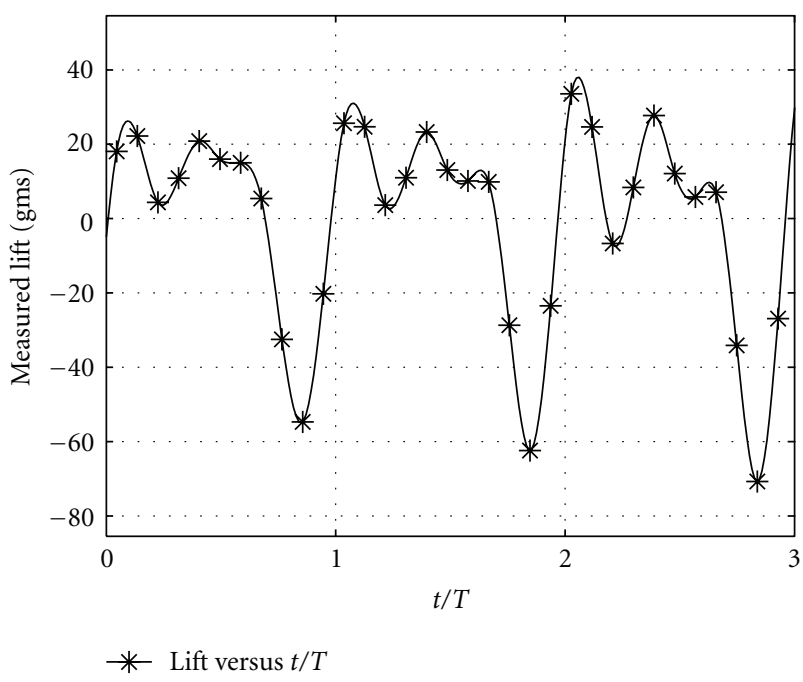

FIGURE 6: Variation of lift force with flapping cycle of elliptic wing $(a=6.35, b=3.175)$ at $f=7.5 \mathrm{~Hz}$.

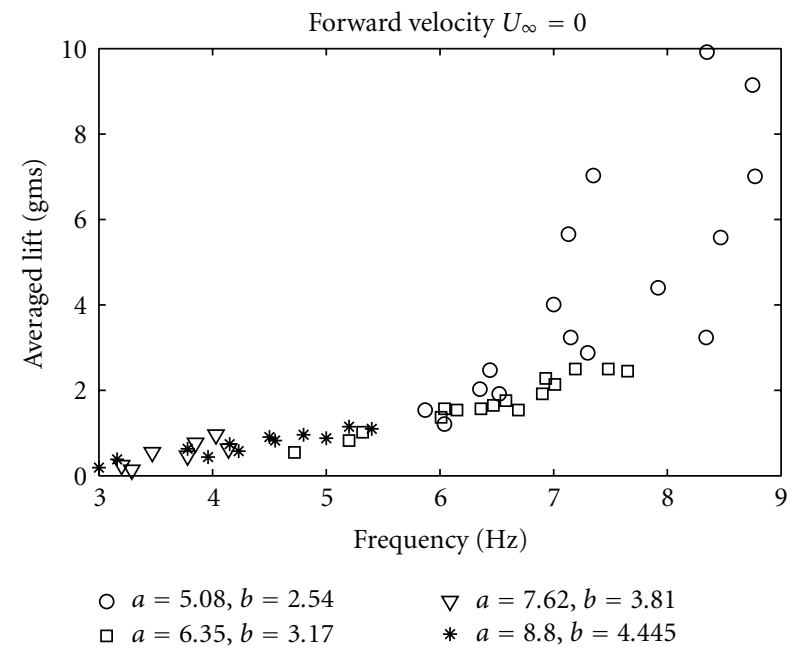

FIgURE 7: Variation of lift versus frequency for elliptical wings of various sizes.

the forward velocity only the added mass term stays which is directly a function of flapping frequency. Therefore at $J=0$ the deviation is more. Whereas with increase in forward velocity and increase in advance ratio the circulatory term also comes in play, the effect of which minimizes the dependence of lift on frequency alone. This trend of lift coefficient versus. advance ratio is found to be similar for the other wings as well. Only two of the wing results are shown to indicate that the change in wing does not shift lift forces much at higher advance ratio, but the deviation will be more at very low and near zero advance ratios only.

7.2. Flow Visualization Results. Extensive visualization experiments are carried out to understand the complex unsteady flow structures during flapping motion. Periodic clap and fling motion of the wing (shown in Figure 5) is associated

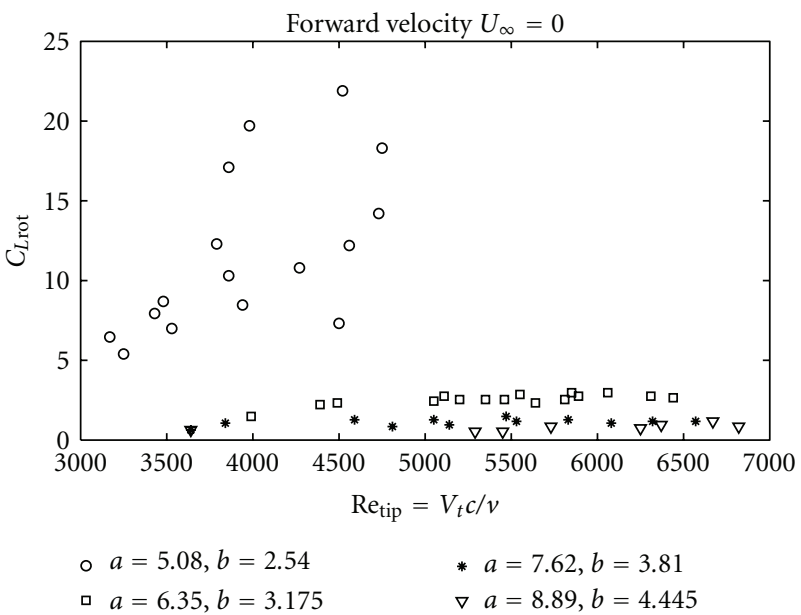

FIGURE 8: Variation of rotational lift coefficient versus Reynolds number $\left(\mathrm{Re}_{\text {tip }}\right)$ for all wing sizes.

with vortex formation around the entire wing periphery and ejection of vortex rings. However, the choice of the wing planform restricts the size of the vortex near the root of the wing which is nearer to the free-surface of the water. Hence, for flow visualization the wing shape chosen is different from that for force measurements. These experiments illustrate the dynamic behavior of the leading edge vortex (LEV).

Figure 10 shows the flow visualization results for the wing shown in Figure 5(a) for $f=0.46 \mathrm{~Hz}$. Wing is started from a position $10^{\circ}$ away from complete downstroke (Figure 5(b)). In Figure 10(a) the motion has just started towards left (marked as face A) which is downstroke. A leading edge vortex has rolled up on the leading edge of face $\mathrm{B}$ as expected. The vortex has grown with time as can be seen in the subsequent pictures (Figures 10(b) and 10(c)). In Figure 10(d), wing has just started moving towards right which is the upstroke motion. As soon as the wing starts moving in an opposite direction, a small vortex is formed on the face $\mathrm{A}$ of the wing. The interaction of these two vortices forms a mushroom shaped vortex pair Figure 10(f) which is then ejected at an angle. The LEV and interaction between these two vortex rings formed around the wing, and its movement in an angular direction with respect to the wing is mainly responsible for the unusually high lift generated by insects (butterflies). The angle of this ejection of the vortex pair determines the contribution of the force generated to lift and thrust. In Figure 10(i), breakdown of the ejected vortex structure has been observed. However, a portion of ejected vortex pair has rolled over to the other side of the wing (Figures 10(h) and 10(i)) and is being dragged along in the upstroke motion. Since the wing is reutilizing this vortex which is created in an earlier stroke, this sustains the lift even in the upstroke. The size of the vortex formed in face A of the wing during upstroke increases as seen in Figures $10(\mathrm{i}), 10(\mathrm{j})$ and $10(\mathrm{k})$.

Same phenomena are also observed when instead of a single-wing a pair of wing is mounted on both the linkages of the flapping model suggesting that the vortex 


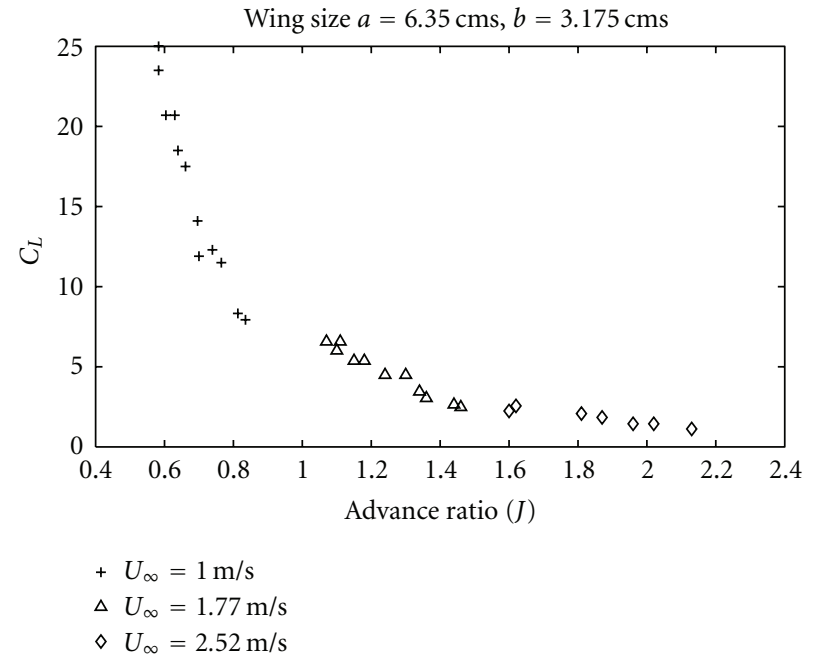

(a)

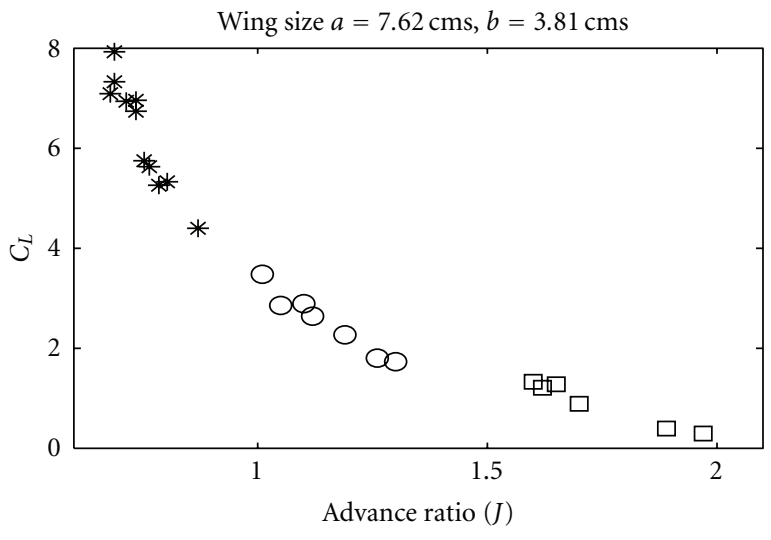
$\square U_{\infty}=2$
$\bigcirc U_{\infty}=1.5$
* $U_{\infty}=1$

Figure 9: Variation of lift coefficient $\left(C_{L}\right)$ versus advance ratio $(J)$ for wing size (a) for elliptical wing $(a=6.35 \mathrm{cms}, b=3.175 \mathrm{cms})$, (b) for elliptical wing $(a=7.61 \mathrm{cms}, b=3.81)$.

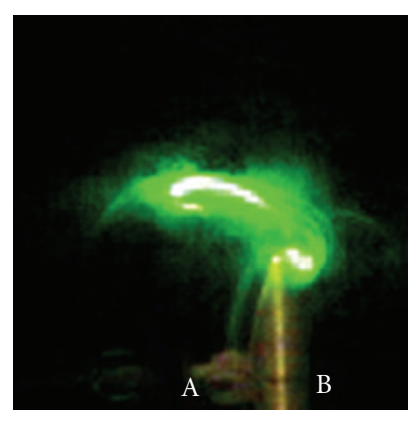

(a) $t=0.08$

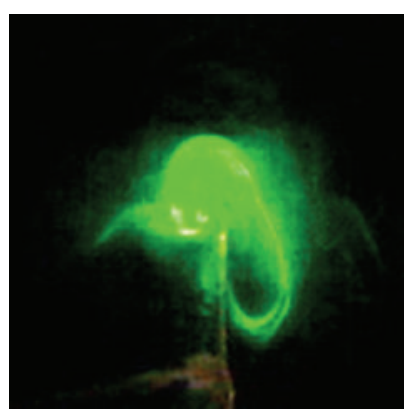

(e) $t=0.48 \mathrm{~s}$

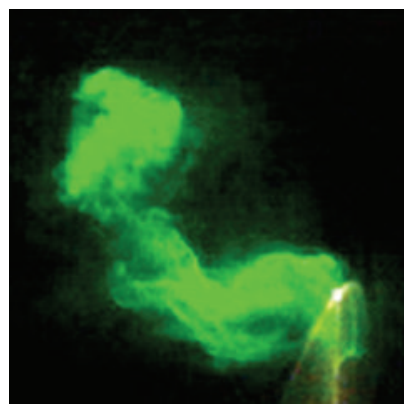

(i) $t=1.26 \mathrm{~s}$

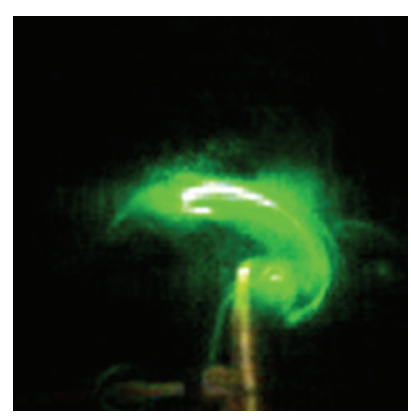

(b) $t=0.12 \mathrm{~s}$

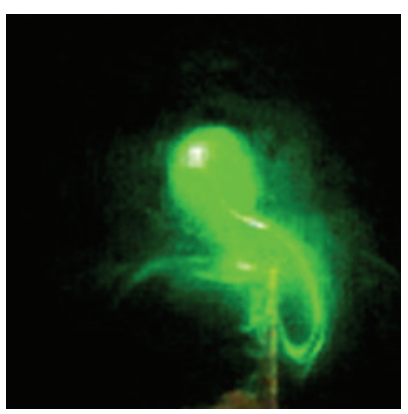

(f) $t=0.6 \mathrm{~s}$

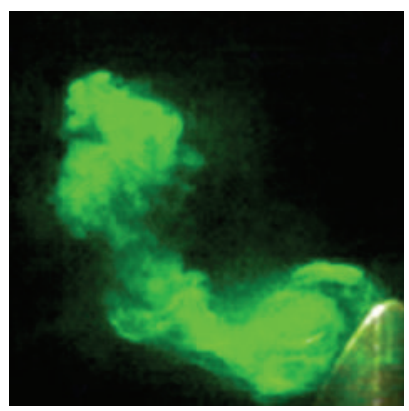

(j) $t=1.38 \mathrm{~s}$

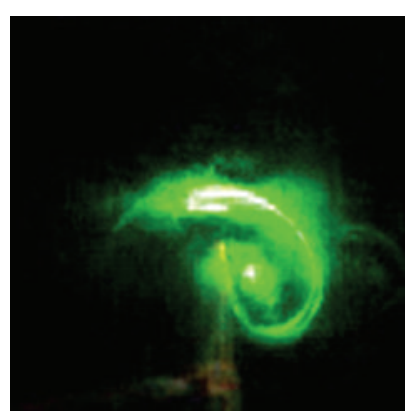

(c) $t=0.24 \mathrm{~s}$

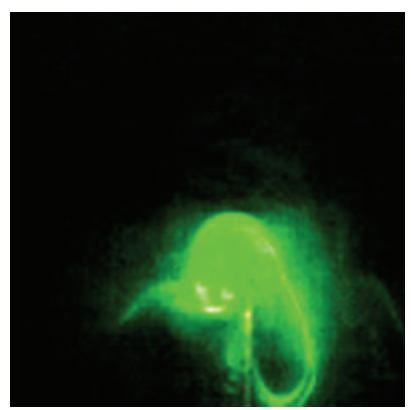

(g) $t=0.84 \mathrm{~s}$

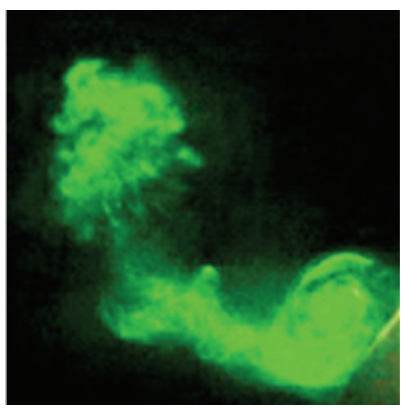

(k) $t=1.5 \mathrm{~s}$

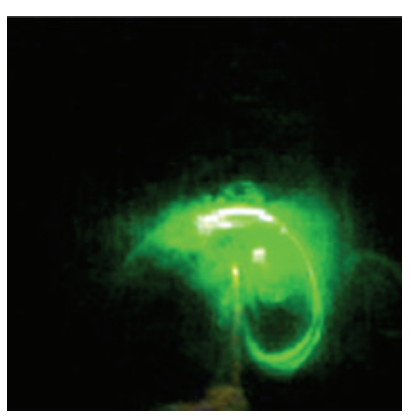

(d) $t=0.36 \mathrm{~s}$

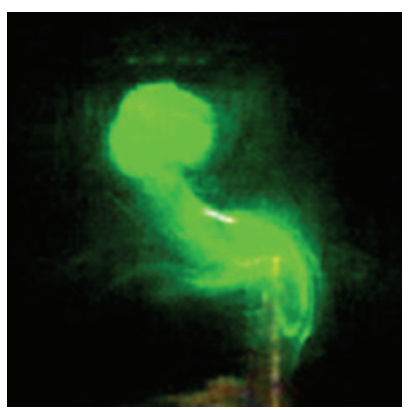

(h) $t=1.02 \mathrm{~s}$

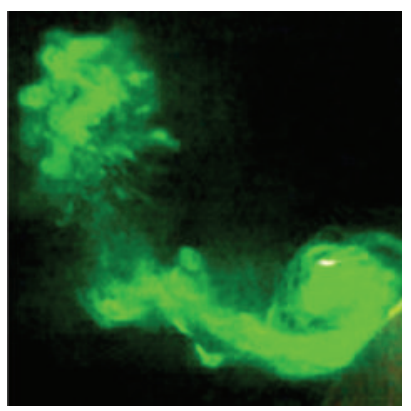

(l) $t=1.62 \mathrm{~s}$

Figure 10: Flow visualization series $(f=0.46 \mathrm{~Hz}$, symmetric single-wing, span $=14 \mathrm{cms})$. 


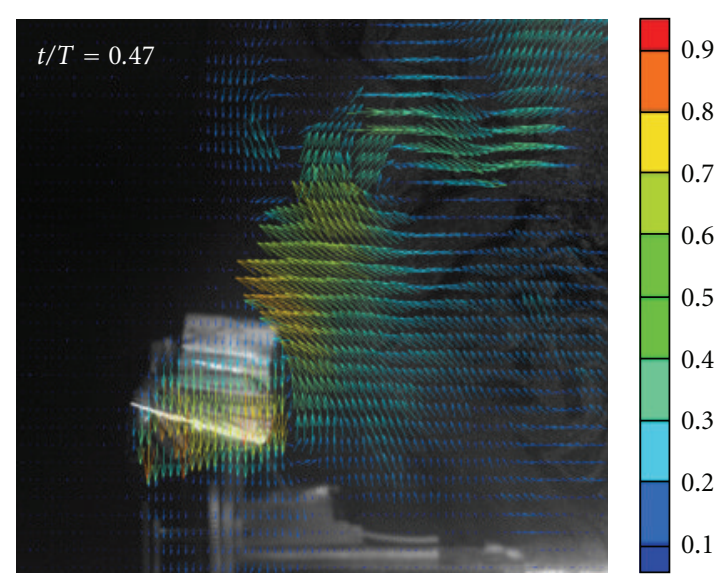

(a)

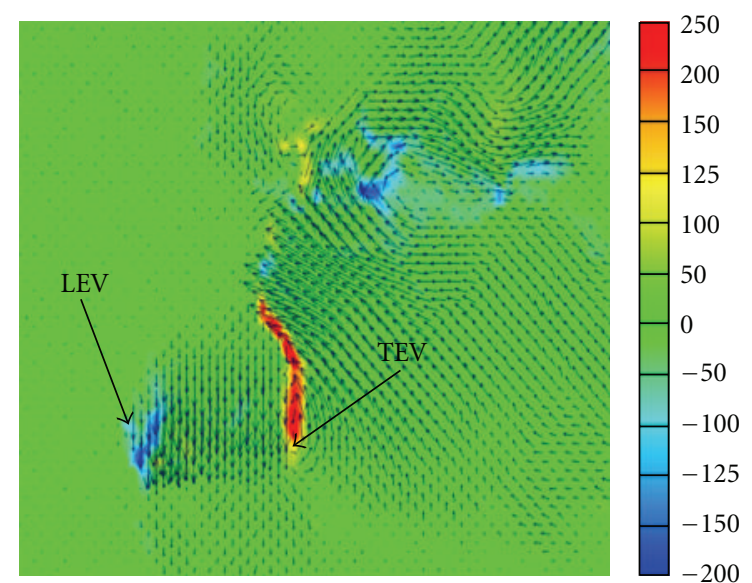

(b)

FIgURE 11: LEV and TEV towards the end of downstroke $(\operatorname{Re}=3768)$.

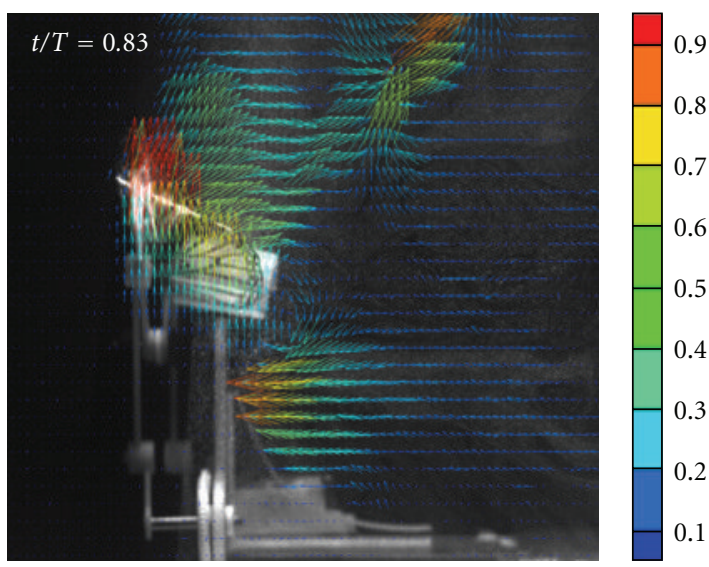

(a)

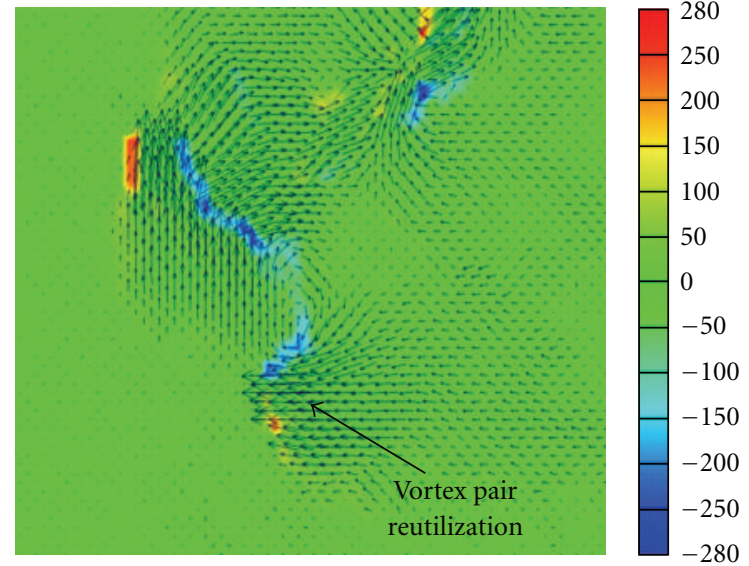

(b)

Figure 12: Vortex reutilization towards the end of upstroke $(\mathrm{Re}=3768)$.

dominated flow features are a common phenomena for flapping wing-type flight provided they are in the same Re range. The timing and levels of interaction and behavior of these vortex pairs do depend on the wing material and its flexibility.

7.3. PIV Results. The PIV experiments are performed on the butterfly shape wing (Figure $4(\mathrm{~b})$ ) at $6 \mathrm{~Hz}$ at different chord cross-sections. All the experiments are carried out at zero forward velocity $(J=0)$, which implies that the study regime is completely unsteady. The effects of underlying vortices and their subsequent interactions in both the strokes have been observed. Similar flow features as seen in the earlier flow visualization is observed in the PIV results as well. Figures 11 and 12 shows the velocity and vorticity fields at $0.5 \mathrm{R}$ of the butterfly wing at $6 \mathrm{~Hz}$ flapping frequency $(\mathrm{Re}=3768)$. The PIV results are shown in two parts as image superimposed with velocity with legends indicating the magnitude of velocity and as velocity superimposed with vorticity contours with the legends showing the magnitude of vorticity. The red levels of vorticity indicate anticlockwise rotation whereas the blue levels indicate the clockwise rotation.

The vortex shown in the flow visualization and the PIV results is in fact three dimensional. The same can be realized by taking various cross-sections along the chord and span; it can be seen that there is a single vortex which connects the whole periphery of the wing. The same has been shown by many in the literature [2-7].

\section{Conclusions}

The variation of forces and flow field with wing size and frequency is studied. The general mechanisms of LEV formation, its growth, its decay in the downstroke, the vortex pair ejection at the stroke reversal as a result of the vortex interactions, vortices growing of the opposite nature in the upstroke, and the subsequent vortex capture are well identified in Figure 10. Similar nature is also observed in the PIV result suggesting that the vortex is in the form of a ring following the peripheral contour of the wing. The source 
and mechanism of generation of lift and its behavior and response to the parameters like frequency and wing sizes are observed. Force results show the dependence on the frequency is more prominent (Figure 7). Even for the same Reynolds number the smaller wing, having higher frequency have more coefficient of lift and vice versa as seen from Figure 8. With increase in advance ratio or with approach from unsteady to quasisteady regime the lift coefficient is found to decline. The strength of ejection does depend on feathering angle as well as the interacting vortices' strength which in turn here depends on the size of the wing (chord) and the tip velocity $\left(V_{t}\right)$ and thus mainly on the Reynolds number.

\section{Nomenclature}

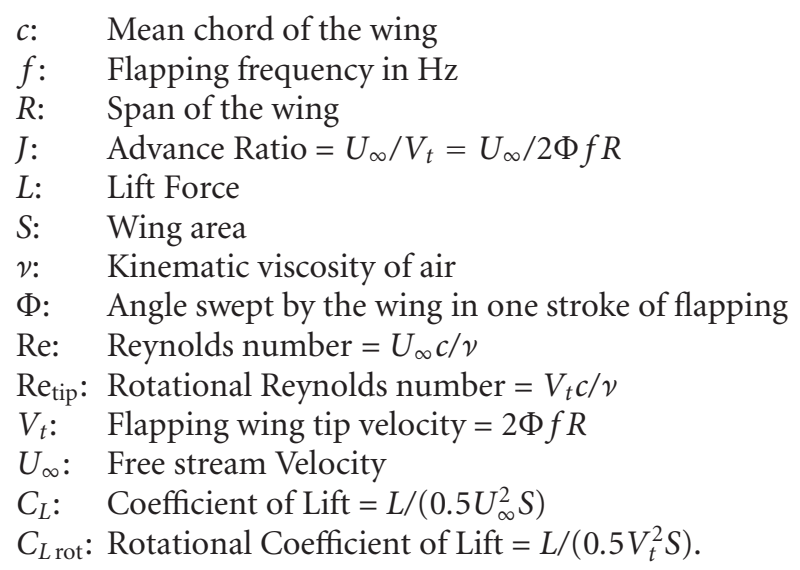

\section{Acknowledgments}

The authors acknowledge the partial financial support from AOARD, AFOSR for the partial support of the work. The authors would also like to acknowledge DST, India for the support for TR-PIV system under FIST.

\section{References}

[1] P. J. Phlips, R. A. East, and N. H. Pratt, "An unsteady lifting line theory of flapping wings with application to the forward flight of birds," Journal of Fluid Mechanics, vol. 112, pp. 97$125,1981$.

[2] D. Lentink and M. H. Dickinson, "Biofluiddynamic scaling of flapping, spinning and translating fins and wings," The Journal of Experimental Biology, vol. 212, no. 16, pp. 2691-2704, 2009.

[3] R. B. Srygley and A. L. R. Thomas, "Unconventional liftgenerating mechanisms in free-flying butterflies," Nature, vol. 420, no. 6916, pp. 660-664, 2002.

[4] R. J. Bomphrey, N. J. Lawson, N. J. Harding, G. K. Taylor, and A. L. R. Thomas, "The aerodynamics of Manduca sexta: digital particle image velocimetry analysis of the leading-edge vortex," The Journal of Experimental Biology, vol. 208, no. 6, pp. 1079-1094, 2005.

[5] C. P. Ellington, "The novel aerodynamics of insect flight: applications to micro-air vehicles," The Journal of Experimental Biology, vol. 202, no. 23, pp. 3439-3448, 1999.

[6] M. H. Dickinson, F. O. Lehmann, and S. P. Sane, "Wing rotation and the aerodynamic basis of insect right," Science, vol. 284, no. 5422, pp. 1954-1960, 1999.
[7] S. P. Sane, "The aerodynamics of insect flight," The Journal of Experimental Biology, vol. 206, no. 23, pp. 4191-4208, 2003.

[8] C. P. Ellington, C. D. van Berg, A. P. Willmott, and A. L. R. Thomas, "Leading-edge vortices in insect flight," Nature, vol. 384, no. 6610, pp. 626-630, 1996.

[9] S. Ho, H. Nassef, N. Pornsinsirirak, Y. C. Tai, and C. M. Ho, "Unsteady aerodynamics and flow control for flapping wing flyers," Progress in Aerospace Sciences, vol. 39, no. 8, pp. 635681, 2003.

[10] T. L. Hedrick, B. W. Tobalske, and A. A. Biewener, "Estimates of circulation and gait change based on a three-dimensional kinematic analysis of flight in cockatiels (Nymphicus hollandicus) and ringed turtle-doves (Streptopelia risoria)," The Journal of Experimental Biology, vol. 205, no. 10, pp. 13891409, 2002.

[11] L. Zhao, Q. Huang, X. Deng, and S. P. Sane, "Aerodynamic effects of flexibility in flapping wings," Journal of the Royal Society Interface, vol. 7, no. 44, pp. 485-497, 2010.

[12] C. P. Ellington, "Aerodynamics and the origin of flight," Advances in Insect Physiology, vol. 23, pp. 171-210, 1991.

[13] M. H. Dickinson and K. G. Gotz, "Unsteady aerodynamic performance of model wings at low Reynolds number," The Journal of Experimental Biology, vol. 174, pp. 45-64, 1993.

[14] D. Lentink and M. H. Dickinson, "Rotational accelerations stabilize leading edge vortices on revolving fly wings," The Journal of Experimental Biology, vol. 212, no. 16, pp. 27052719, 2009.

[15] D. Lentink, F. T. Muijres, F. J. Donker-Duyvis, and J. L. van Leeuwen, "Vortex-wake interactions of a flapping foil that models animal swimming and flight," The Journal of Experimental Biology, vol. 211, no. 2, pp. 267-273, 2008.

[16] S. P. Sane and M. H. Dickinson, "The control of flight force by a flapping wing: lift and drag production," The Journal of Experimental Biology, vol. 204, no. 15, pp. 2607-2626, 2001.

[17] J. M. Birch and M. H. Dickinson, "Spanwise flow and the attachment of the leading-edge vortex on insect wings," Nature, vol. 412, no. 6848, pp. 729-733, 2001. 

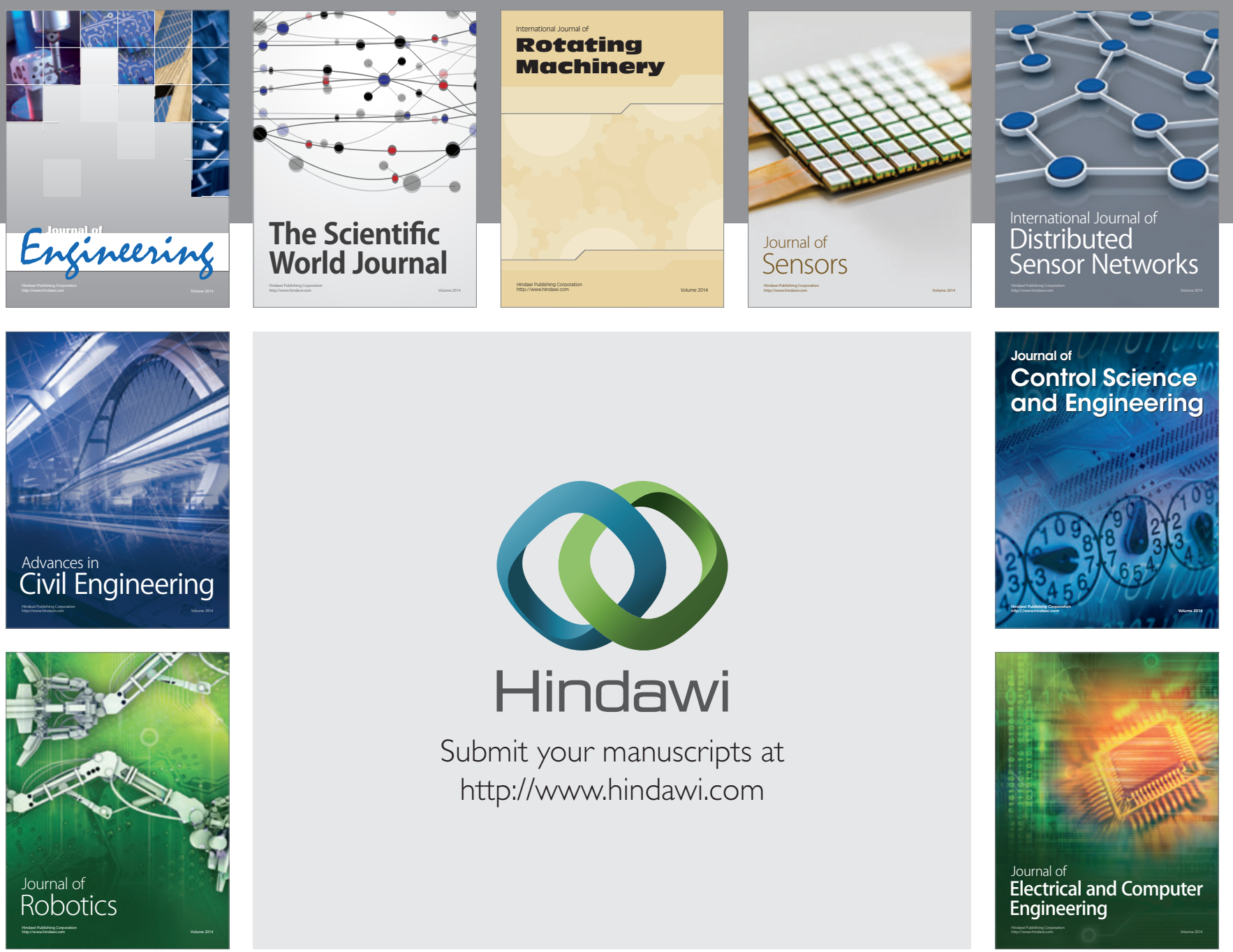

Submit your manuscripts at

http://www.hindawi.com
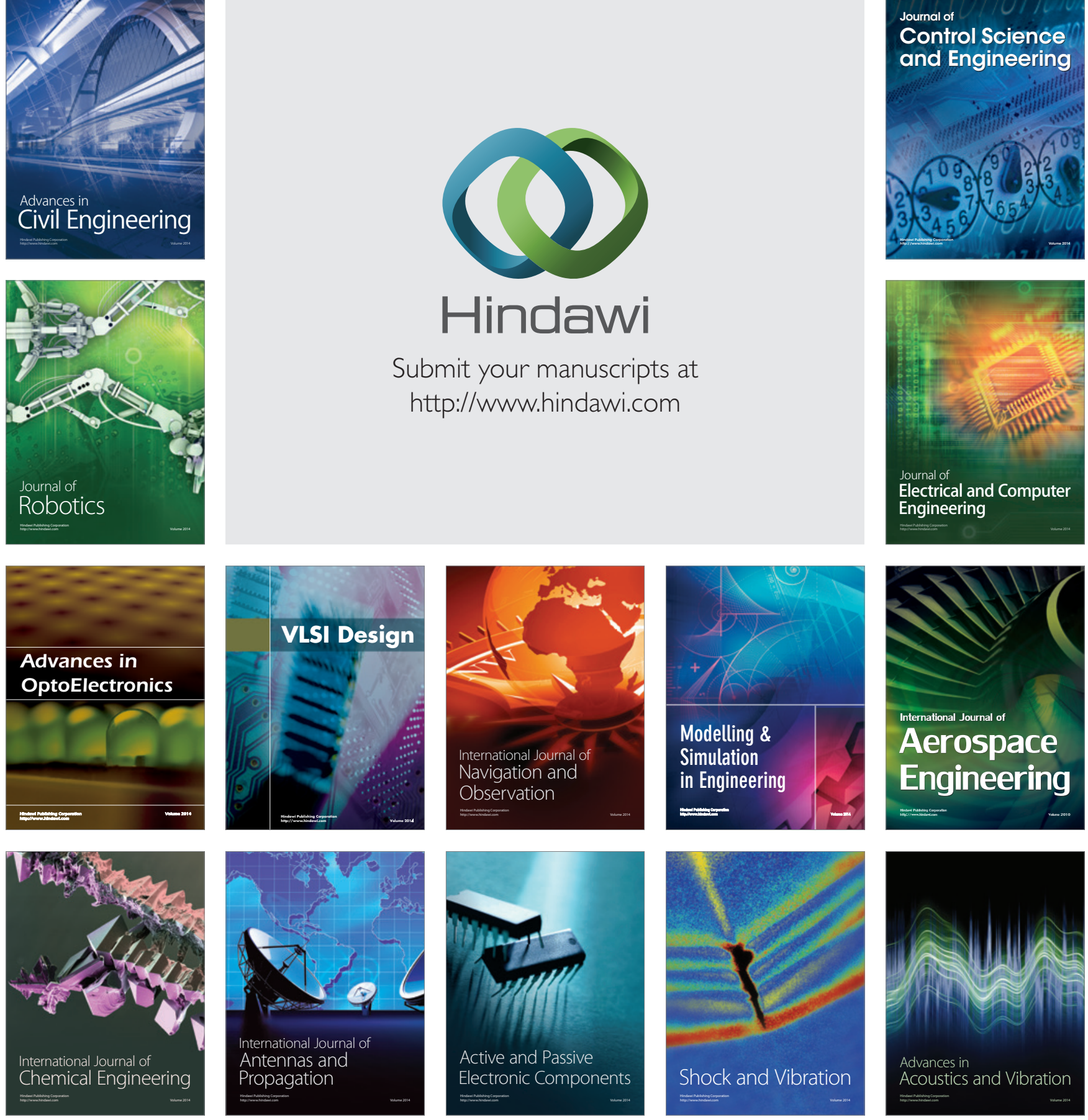